Phys. Rev. E., March, 2001

\title{
Universal scaling functions for bond percolation on planar random and square lattices with multiple percolating clusters
}

\author{
Hsiao-Ping Hsu ${ }^{1, *}$, Simon C. Lin ${ }^{1,2}$,Chin-Kun $\mathrm{Hu}^{2,3,+}$ \\ ${ }^{1}$ Computing Centre, Academia Sinica, Nankang, Taipei 11529, Taiwan \\ ${ }^{2}$ Institute of Physics, Academia Sinica, Nankang, Taipei 11529, Taiwan \\ 3 Department of Physics, National Dong Hwa University, Hualien 97401, Taiwan
}

(Dated: November 17, 2018)

\begin{abstract}
Percolation models with multiple percolating clusters have attracted much attention in recent years. Here we use Monte Carlo simulations to study bond percolation on $L_{1} \times L_{2}$ planar random lattices, duals of random lattices, and square lattices with free and periodic boundary conditions, in vertical and horizontal directions, respectively, and with various aspect ratio $L_{1} / L_{2}$. We calculate the probability for the appearance of $n$ percolating clusters, $W_{n}$, the percolating probabilities, $P$, the average fraction of lattice bonds (sites) in the percolating clusters, $\left\langle c^{b}>_{n}\left(<c^{s}>_{n}\right)\right.$, and the probability distribution function for the fraction $c$ of lattice bonds (sites), in percolating clusters of subgraphs with $n$ percolating clusters, $f_{n}\left(c^{b}\right)\left(f_{n}\left(c^{s}\right)\right)$. Using a small number of nonuniversal metric factors, we find that $W_{n}, P,<c^{b}>_{n}\left(<c^{s}>_{n}\right)$, and $f_{n}\left(c^{b}\right)\left(f_{n}\left(c^{s}\right)\right)$ for random lattices, duals of random lattices, and square lattices have the same universal finite-size scaling functions. We also find that nonuniversal metric factors are independent of boundary conditions and aspect ratios.

PACS numbers: 05.50.+q, 64.60.Ak, 75.10.-b
\end{abstract}




\section{INTRODUCTION}

Percolation is related to many interesting scientific phenomena [1]. In recent years percolation problems with multiple percolating clusters have attracted much attention [2-19]. Most of the simulational studies of such problems have been restricted to percolation on lattices [20]. However, many physical systems with multiple percolating clusters such as Carbino disks used in the study of quantum Hall effects [2], or oil fields confronted with drilling problems, do not have underlined regular lattice structures. Thus, it is of interest to know the relationship between the quantities for percolation on regular lattices and the quantities for percolation not on regular lattices, such as random lattices. In the present paper, we use Monte Carlo simulations to study bond percolation on $L_{1} \times L_{2}$ planar random lattices, duals of random lattices, and square lattices with free and periodic boundary conditions in vertical and horizontal directions, respectively, and with various aspect ratio $L_{1} / L_{2}$. We calculate the probability for the appearance of $n$ percolating clusters, $W_{n}$, the percolating probabilities, $P$, the average fraction of lattice bonds (sites) in percolating clusters, $\left\langle c^{b}\right\rangle_{n}$ $\left(<c^{s}>_{n}\right)$, and the probability distribution function for fraction $c$ of lattice bonds (sites), in percolating clusters of subgraphs with $n$ percolating clusters, $f_{n}\left(c^{b}\right)\left(f_{n}\left(c^{s}\right)\right)$. Using a small number of nonuniversal metric factors, we find that $W_{n}, P,\left\langle c^{b}>_{n}\left(<c^{s}>_{n}\right)\right.$, and $f_{n}\left(c^{b}\right)\left(f_{n}\left(c^{s}\right)\right)$ for random lattices, duals of random lattices, and square lattices, have the same universal finite-size scaling functions. We also find that nonuniversal metric factors are independent of boundary conditions and aspect ratios. Furthermore, this study is related to recent developments in the universality and scaling of critical phenomena.

Universality and scaling are two important concepts in modern theory of critical phenomena [21, 22, 23], and percolation models are an ideal system for studying critical phenomena [1]. Thus, universality and scaling have been actively studied in recent decades, especially for percolation models [24]. In 1992, Langlands et. al. 25] proposed that for bond and site percolation models on square (sq), planar triangular (pt), and honeycomb (hc) lattices, the critical existence probability (also called crossing probability or spanning probability) is a universal quantity, when aspect ratios of sq, pt, and hc lattices have relative ratios, $1: \sqrt{3}$ : $\sqrt{3} / 2$. From 1995 1996, Hu, Lin and Chen (HLC) [3], 26] calculated existence probability, $E_{p}$, percolation probability, $P$, and probability for the appearance of $n$ percolating clusters, $W_{n}$, of bond and site percolation models, on sq, hc, and pt lattices with aspect ratios, 
$1: \sqrt{3}: \sqrt{3} / 2$, and showed that all their scaled data fall on the same universal scaling functions, by selecting a very small numbers of nonuniversal metric factors, and maintaining similar nonuniversal metric factors under two boundary conditions, free and periodic boundary conditions. By using renormalization group theory, Hovi and Aharony in 1996 [27] also pointed out that scaling functions, for the spanning probability are universal at the fixed point for every system with the same dimensionality, spanning rule, aspect ratio and boundary conditions. Okabe and Kikuchi in 1996 [28], extended the work of HLC to a two-dimensional Ising model on planar regular lattices. In 1997, Hu and Wang [11 found that the lattice percolation and continuum percolation of hard and soft disks, have the same universal scaling functions for $W_{n}$. Using the connection between an Ising model and a bond-correlated percolation model [29], Tomita, Okabe, and $\mathrm{Hu}$ in 1999 [17], calculated the probability for the appearance of $n$ percolating clusters, $W_{n}$, the percolating probabilities, $P$, the average fraction of lattice sites in percolating clusters, $\langle c\rangle_{n}$, and the probability distribution function for the fraction $c$ of lattice sites in percolating clusters of subgraphs with $n$ percolating clusters, $f_{n}(c)$, for bond-correlated percolation model on sq, hc, and pt lattices, with aspect ratios of $1: \sqrt{3}: \sqrt{3} / 2$. Using a small number of nonuniversal metric factors, they found that $W_{n}, P,\left\langle c>_{n}\right.$, and $f_{n}(c)$ for sq, hc, and pt lattices have the same universal finite-size scaling functions.

However, the studies mentioned above are mostly focused on regular lattices, with fixed coordination numbers [20]. In $1999 \mathrm{Hsu}$ and Huang (HH) [30] determined the percolation thresholds and critical exponents, and demonstrated explicitly that the ideas of universal critical exponents and universal scaling function with nonuniversal metric factors can be extended to bond percolation on $L \times L$ periodic planar random lattices, duals of random lattices, and square lattices, for both existence and percolating probabilities, and mean cluster size. This paper will study bond percolation on $L_{1} \times L_{2}$ planar random lattices, duals of random lattices, and square lattices, in more detail, and consider the case that the lattices have free and periodic boundary conditions, in vertical and horizontal directions, respectively, as in [3]. Percolating probability is defined by the ratio of the number of bonds in the percolating clusters to the total number of bonds in 30. Here, we consider two different definitions of the percolating probability, in terms of bonds and in terms of sites; the latter was also used in [3] and [17]. We calculate the probability $W_{n}$ for the appearance of $n$ percolating clusters, the percolating probability $P$, the average fraction 
of lattice bonds (sites) in percolating clusters, $\left\langle c^{b}>_{n}\left(<c^{s}>_{n}\right)\right.$, and the probability distribution function for the fraction $c$ of lattice bonds (sites) in percolating clusters of subgraphs with $n$ percolating clusters, $f_{n}\left(c^{b}\right)\left(f_{n}\left(c^{s}\right)\right)$, for various values of aspect ratios $L_{1} / L_{2}$, and finally check the universal finite-size scaling behaviors for these quantities. In [30], HH used two nonuniversal metric factors, $D_{2}$ and $D_{3}$, to fix universal finite-size scaling functions, for percolating probability in terms of bonds. In the present paper, we calculated two nonuniversal metric factors of percolating probability, in terms of sites and obtained previous known values of nonuniversal metric factors determined by HH, to check whether we could have universal scaling functions for $W_{n}, P,<c^{b}>_{n}\left(<c^{s}>_{n}\right)$, and $f_{n}\left(c^{b}\right)\left(f_{n}\left(c^{s}\right)\right)$, of bond percolation on random lattices, duals of random lattices, and square lattices.

Dirichlet and Voronoi [31] first used the concept of random lattices in condensed matter theory and Christ, Friedberg, and Lee (CFL) [32] used another type of random lattices to formulate quantum field theory. Here, we adopt the CFL algorithm and give a brief review of the construction of planar random lattices and their duals. First, we randomly generate $N$ sites in the $L_{1} \times L_{2}$ rectangular domain with periodic boundary conditions. Next, we choose three nearby sites arbitrarily, and draw a circle to go through the three sites. If there are no lattice sites inside the circle, the three sites are connected by links to form a triangle. A planar random lattice is constructed by repeating the process until all sites are connected by links. The whole rectangular domain is divided into $2 N$ non-overlapping triangles, whose vertices are sites of the random lattice, and circle centers with triangles are the sites of dual lattice. Thus, there is a one to one correspondence between triangles and dual lattice sites. Because a link of the random lattice is shared by two triangles, the two corresponding dual lattice sites are connected by one dual link. There is a one to one correspondence between links and dual links. The whole rectangular domain is partitioned into $N$ non-overlapping planar convex polyhedra, which are formed by dual links and the vertices of $N$ polyhedra are sites for dual lattice. There is also a one to one correspondence between the lattice sites and polyhedra on dual lattice. Examples of a planar random lattice with its dual, under periodic boundary conditions, in both vertical and horizontal directions, are shown in Fig. $1(\mathrm{a})$.

This paper is organized as follows: In Sec. II, we present the simulational results for $W_{n}, P,<c^{b}>_{n}\left(<c^{s}>_{n}\right)$, and $f_{n}\left(c^{b}\right)\left(f_{n}\left(c^{s}\right)\right)$ for bond percolation, on $L_{1} \times L_{2}$ random lattices, duals of random lattices, and square lattices, under free and periodic boundary 
conditions in vertical and horizontal directions with $L_{1} / L_{2}=4$. The boundary bonds which cross the rectangular domain in vertical direction on random lattices, due to periodic boundary conditions, are eliminated because of free boundary conditions in vertical direction considered in this paper. We adopt the method of $\mathrm{HH}$ [30], to find percolating clusters. Only the first kind of percolating cluster paths, without boundary bonds in the vertical direction (the clusters extend from top to bottom), should be identified, and an example of this is shown in Fig. 1(b). In Sec. III, we use finite-size scaling theory to check the scaling behaviors of various quantities, and to show that such quantities have universal finite-size scaling functions for regular lattices and random lattices. A summary is provided in Sec. IV.

II. $W_{n}\left(L_{1}, L_{2}, p\right), f_{n}(c)$, and $<c>_{n}$

We see the bond percolation on a lattice $G$, with linear dimensions $L_{1}$ and $L_{2}$ in horizontal and vertical directions, respectively; the probability for the appearance of $n$ top-to-bottom percolating clusters, $W_{n}\left(L_{1}, L_{2}, p\right)$, is defined by [3]

$$
W_{n}\left(L_{1}, L_{2}, p\right)=\sum_{G_{n}^{\prime} \subseteq G} p^{b\left(G_{n}^{\prime}\right)}(1-p)^{E-b\left(G_{n}^{\prime}\right)} .
$$

Here, the percolating cluster is defined as a cluster extending from top to bottom in $G, G_{n}^{\prime}$ denotes a percolating subgraph with $n$ percolating clusters, $b\left(G_{n}^{\prime}\right)$ is the number of occupied bonds in $G_{n}^{\prime}$, and $E$ is the total number of links in $G$. The existence probability $E_{p}$ can be expressed obviously as

$$
E_{p}=\sum_{n=1}^{\infty} W_{n}
$$

with $W_{0}=1-E_{p}$.

To obtain more detailed information about the contents of the percolating cluster, following Tomita et. al. [17], we decompose $W_{n}$ as

$$
W_{n}=\int_{0}^{1} f_{n}(c) d c
$$

where $n=1, \ldots \infty, c$ denotes the fraction of lattice bonds (sites) in percolating clusters, and $f_{n}(c)$ is the probability distribution function of $c$ in subgraphs with $n$ percolating clusters. 
The probability distribution function of $c$ in all subgraphs is the overall summations of $f_{n}(c)$, i.e.,

$$
f(c)=\sum_{n=1}^{\infty} f_{n}(c)
$$

In terms of $f_{n}(c)$, the average fraction of lattice bonds (sites) in subgraphs with $n$ percolating clusters can be expressed as

$$
<c>_{n}=\int_{0}^{1} c f_{n}(c) d c,
$$

where $n=1, \ldots, \infty$, and percolating probability $P$ can be written as

$$
<c>=\sum_{n=1}^{\infty}<c>_{n}=\int_{0}^{\infty} c f(c) d c=P .
$$

To generate subgraphs, we use the random bond occupation process with equal occupation probability for each link. The simulations are performed on $128 \times 32,256 \times 64$, and $512 \times 128$ planar random (pran) lattices, and their duals (dpran), with free and periodic boundary conditions in the vertical and horizontal directions, respectively. To compare the results with regular lattices, we also perform simulations on square (sq) lattices of the same sizes. On each lattice, we take 60 occupation probabilities around the critical percolation threshold for every 0.002 increment, and use the random bond occupation process to generate $10^{5} \sim 10^{6}$ configurations for each occupied probability, $p$. We calculate $W_{n}\left(L_{1}, L_{2}, p\right),\left\langle c^{b}>_{n}\right.$, and $<c^{s}>_{n}$, where $c^{b}$ denotes the fraction of bonds in percolating clusters, and $c^{s}$ denotes the fraction of sites in percolating clusters; and the results are shown in Figs. 2 and 3. The calculated results of the percolating probabilities in terms of bonds, $P^{b}$, and in terms of sites, $P^{s}$, are also shown in Fig. 3. We calculate $f_{n}\left(c^{b}\right)$ and $f_{n}\left(c^{s}\right)$ at $p=p_{c}$ and take $p_{c}=0.3333$ for planar random lattices, and $p_{c}=0.6667$ for dual lattice [30. The results are shown in Fig. 4. The differences between bond and site contents in percolating clusters are shown in Figs. 3 and 4; here, for the clarity of presentation only, the results for $512 \times 128$ lattices are plotted in the figures.

\section{UNIVERSAL FINITE-SIZE SCALING FUNCTIONS}

The finite-size scaling theory was first formulated by Fisher in 1971 [22]. According to the theory, for a physical quantity $X$, which scales as $X(t) \sim t^{\rho}$ in a thermodynamic system 
near critical point $t=0$, then the same quantity in a finite system with linear dimension $L$, $X_{L}(t)$, should obey the general law,

$$
X_{L}(t) \sim L^{-\rho / \nu} F\left(t L^{1 / \nu}\right)
$$

Here, $F(x)$ with $x=t L^{1 / \nu}$ labelled as scaling function, with $\nu$ as correlation length exponent. In 1984, Privman and Fisher [23] considered universal finite-size scaling functions and nonuniversal metric factors, and proposed that the singular part of the free energy of a critical system can be written as

$$
f_{L}^{s}(t) \sim L^{-d} Y\left(D t L^{1 / \nu}\right)
$$

where $d$ is the spatial dimensionality of lattice, $Y$ is a universal scaling function, and $D$ is a nonuniversal metric factor.

At the critical point, $p=p_{c}$, there also exists a finite-size scaling form for the distribution function of $X_{L}(t)[17]$ :

$$
Q\left(X_{L}(t=0)\right) \sim L^{\rho / \nu} Y\left(X_{L}(t=0) \cdot L^{\rho / \nu}\right) .
$$

In [26, 30], three nonuniversal metric factors $D_{1}, D_{2}$, and $D_{3}$ were used for regular lattices and random lattices, to describe the universal scaling functions of existence probability $E_{p}$, and the percolating probability $P$, i.e.

$$
E_{p}(p, L)=F(x)
$$

with $x=D_{1}\left(p-p_{c)} L^{1 / \nu}\right.$ and

$$
D_{3} P(p, L)=L^{-\beta / \nu} S_{p}(z)
$$

with $z=D_{2}\left(p-p_{c}\right) L^{1 / \nu}$.

Following $\mathrm{Hu}$ et. al. [3], we use the evaluated percolation thresholds $p_{c}$ [30], and the exact values of critical exponent $\nu=4 / 3$, to plot $W_{n}$ as a function of $y=\left(p-p_{c}\right) L^{1 / \nu}$, for planar random lattices and their duals in Figs. 5(a) and 5(b), respectively. We can see from these results, that the scaled data for $W_{n}$ can be described by a single scaling function $F_{n}(r, y)$ with $r=L_{1} / L_{2}$, and $F_{n}(r, y)$ for $n \geq 2$ as a symmetric function of $y$. In Fig. 6 we plot $W_{n}\left(L_{1}, L_{2}, p\right)$ as a function of $x$ for bond percolation on $512 \times 128$ random lattice, dual of random lattice, and square lattice, where $x=D_{1}\left(p-p_{c}\right) L^{1 / \nu}$, with $D_{1}$ taken from 
Table I and $L=\left(L_{1} \times L_{2}\right)^{1 / 2}$. Fig. 6 shows that the calculated results for each $n$ can be well described by a single universal scaling function, $U_{n}(x)$.

In $\mathrm{Hu}$ and Lin's paper [3], the scaling functions $F_{n}(r, y)$ were calculated for bond percolation on square lattice for various values of aspect ratios, $r$. We will examine whether the same nonuniversal metric factors, $D_{1}$, can be extended to different aspect ratios. We calculate $W_{n}$ for $L_{1} \times L_{2}$ random lattices, dual of random lattices, and square lattices with $r=L_{1} / L_{2}=1,2, \ldots, 6$ and determine the universal scaling functions, $U_{n}(r, x)$, where $x=D_{1}\left(p-p_{c}\right) L^{1 / \nu}$ and where $D_{1}$ is taken from Table I. The results for $n=1$ and 2 are shown in Figs. 7(a) and 7(b), respectively. We can see that the scaled data for each $r$ can be described by a single universal scaling function very well. The results of $U_{n}(r, x)$ as a function of $r$ for $n=0,1, \ldots, 4$ at the critical point $p=p_{c}$, are presented in Fig. 8(a) which show that the three lattices provide similar results. We also calculate the average number of percolating clusters $C(r, x)$, defined by

$$
C(r, x)=\sum_{n=1}^{\infty} U_{n}(r, x) n .
$$

$C(r, 0)$ for random lattice, dual of random lattice, and square lattice as a function of $r$ are shown in Fig. 8(b). Fig. 8(b) shows that $C(r, 0)$ increases linearly with an increasingly large, $r$ and that different lattices have the same slope of approximately 0.43.

Tomita et. al. [17] had obtained universal finite-size scaling functions of $\langle c\rangle_{n},\langle c\rangle$, $f_{n}(c)$, and $f(c)$ for a bond-correlated percolation model, corresponding to an Ising model on planar regular lattices. It is of interest to extend such study to bond random percolation on random lattices. From Eqs.(5), (6), and (11), the universal scaling function of $\langle c\rangle$ and $<c>_{n}$ can be expressed as

$$
D_{3}<c(p, L)>=L^{-\beta / \nu} G(z),
$$

and

$$
D_{3}<c(p, L)>_{n}=L^{-\beta / \nu} G_{n}(z),
$$

with $z=D_{2}\left(p-p_{c}\right) L^{1 / \nu}$. At $p=p_{c}$, the universal scaling function of $f(c)$ and $f_{n}(c)$ are expressed as

$$
D_{3}^{-1} f(c)=L^{\beta / \nu} H\left(z^{\prime}\right)
$$


and

$$
D_{3}^{-1} f_{n}(c)=L^{\beta / \nu} H_{n}\left(z^{\prime}\right)
$$

with $z^{\prime}=D_{3} c L^{\beta / \nu}$. To check the finite-size scaling and universality of these quantities, we use simulation results for $256 \times 64$ and $512 \times 128$ square lattices, planar random lattices and their duals. In [30], the percolating probability $P$ is defined in terms of the bond number in percolating clusters, and the nonuniversal metric factors $D_{2}=D_{2}^{b}$ and $D_{3}=D_{3}^{b}$ are used. To evaluate factors $D_{2}^{s}$ and $D_{3}^{s}$, we adopt the same procedure as in [30], plotting $P^{b} / L^{-\beta / \nu}$ and $P^{s} / L^{-\beta / \nu}$ as functions of $y=\left(p-p_{c}\right) L^{1 / \nu}$, as shown in Fig. 9. All of the nonuniversal metric factors for different types of lattices used in this paper are listed in Table I.

We plot $D_{3} P / L^{-\beta / \nu}$ and $D_{3}<c>_{n} / L^{-\beta / \nu}$ as a function of $z=D_{2}\left(p-p_{c}\right) L^{1 / \nu}$, in Figs. 10(a) and 10(b) for bond content and site content, respectively, with $D_{2}$ and $D_{3}$ taken

from Table I. At $p=p_{c}$, the scaled data $D_{3}^{-1} f(c) / L^{\beta / \nu}$, and $D_{3}^{-1} f_{n}(c) / L^{\beta / \nu}$ as functions of $z^{\prime}=D_{3} c L^{\beta / \nu}$, are presented in Figs. 11(a) and 11(b), respectively, for the bond content and site content. Figs. 10 and 11 show that the bond percolation processes on square lattices, random lattices and their duals have universal finite-size scaling functions.

\section{SUMMARY AND DISCUSSION}

Having used nonuniversal metric factors from Table I in this paper, we have found that the universal finite-size scaling functions for $W_{n}$ (Figs. 6 and 7 ), $<c^{b}>_{n}$ and $P^{b}$ (Fig. 10(a)), $\left\langle c^{s}>_{n}\right.$ and $P^{s}$ (Fig. 10(b)), $f_{n}\left(c^{b}\right)$ (Fig. 11(a)), and $f_{n}\left(c^{s}\right)$ (Fig. 11(b)). Fig. 7 includes results for different aspect ratios $r$, i.e. $6 \geq r \geq 1$. The values of nonuniversal metric factors, $D_{1}, D_{2}^{b}$, and $D_{3}^{b}$ of Table I are consistent with the corresponding values of Ref. [30], where the boundary conditions are different from the boundary conditions of the present paper. These results suggest that in random lattices, the nonuniversal metric factors are also independent of the boundary conditions and aspect ratios, as in the case of regular lattices [26]. Please also note that $D_{2}^{s}$ and $D_{3}^{s}$ in Table I are consistent within numerical errors.

Many interesting problems are related to the properties of multiple percolating clusters. It is of interest to extend the study of the present paper to higher spatial dimensions. In particular, the further study of multiple percolating clusters in three dimensions could be 
related to an oil drilling problem.

\section{Acknowledgments}

This work was supported in part by the National Science Council of the Republic of China (Taiwan) under Contract No. NSC 89-2112-M-001-084. 
[*] Electronic address: hphsu@gate.sinica.edu.tw

[+] Electronic address: huck@phys.sinica.edu.tw.

[1] D. Stauffer and A. Aharony, Introduction to Percolation Theory Revised 2nd. ed. (Taylor and Francis, London, 1994).

[2] I. M. Ruzin, N. R. Cooper, B. I. Halperin, Phys. Rev. B. 53, 1558 (1996).

[3] C.-K. Hu and C. Y. Lin, Phys. Rev. Lett. 77, 8 (1996).

[4] P. Sen, Int. J. Modern Phys. C 7, 603 (1996).

[5] C.-K. Hu and B. I. Halperin, Phys. Rev. B 55, 2705 (1997).

[6] D. Stauffer, Physica A 242, 1 (1997).

[7] M. Aizenman, Nucl. Phys. B [FS] 485, 551 (1997).

[8] L. N. Shchur and S. S. Kosyakov, Int. J. Modern Phys. C 8, 473 (1997).

[9] P. Sen, Int. J. Modern Phys. C 8, 229 (1997).

[10] N. R. Cooper, B. I. Halperin, C.-K. Hu, I. M. Ruzin, Phys. Rev. B 55, 4551 (1997).

[11] C.-K. Hu and F. G. Wang, J. Korean Phys. Soc. 31, S271 (1997).

[12] P. Sen and A. Aharony, Int. J. Mod. Phys. C 8, 1169 (1997).

[13] J. L. Cardy, J. Phys. A: Math. Gen. 31, L105 (1998). See also, H. Saleur and B. Duplantier, Phys. Rev. Lett. 58, 2325 (1987).

[14] C.-Y. Lin, C.-K. Hu and J.-A. Chen, J. Phys. A 32, L111 (1998).

[15] C.-Y. Lin and C.-K. Hu, Phys. Rev. E 58, 1521 (1998).

[16] Y. Okabe, K. Kaneda, M. Kikuchi, and C.-K. Hu, Phy. Rev. E 59, 1585 (1999).

[17] Y. Tomita, Y. Okabe, C.-K. Hu, Phy. Rev. E 60, 2716 (1999).

[18] C.-K. Hu, J.-A. Chen and C.-Y. Lin, Physica A 266, 27 (1999).

[19] P. Sen, Int. J. Mod. Phys. C 10, 747 (1999).

[20] One exception is the study of continuum percolation of soft disks and hard disks, see [11].

[21] H. E. Stanley, Introduction to Phase Transitions and Critical Phenomena, (Oxford Univ. Press, New York, 1971).

[22] M. E. Fisher, in Proc. 1970 E. Fermi Int. School of Physics, M. S. Green ed. (Academic, NY, 1971) Vol. 51, P. 1.; M. E. Fisher and M. N. Barber, Phys. Rev. Lett. 28, 1516 (1972).

[23] V. Privman and M. E. Fisher, Phys. Rev. B 30, 322 (1984). 
[24] For a recent review, see Y. Okabe, K. Kaneda, Y. Tomita, M. Kikuchi, C.-K. Hu, Physica A 281, 233 (2000).

[25] R. P. Langlands, C. Pichet, Ph. Pouliot, and Y. Saint-Aubin, J. Stat. Phys. 67, 553 (1992); see also R. M. Ziff, Phys. Rev. Lett 69, 2670 (1992) and R. P. Langlands, Ph. Pouliot, and Y. Saint-Aubin, Bull. Am. Math. Soc. 30, 1 (1994).

[26] C.-K. Hu, C. Y. Lin and J. A. Chen, Phys. Rev. Lett. 75, 193 (1995); 75, 2786E (1995); Physica A 221, 80 (1995).

[27] J.-P Hovi and A. Aharony, Phys. Rev. E 53, 235 (1996).

[28] Y. Okabe and M. Kikuchi, Int. J. Mod. Phys. C 7, 287 (1996).

[29] C.-K. Hu, Phys. Rev. B 29, 5103 and 5109 (1984).

[30] H.-P. Hsu and M.-C. Huang, Phys. Rev. E 60, 6361 (1999).

[31] C. Itzykson and J. M. Drouffe, Statistical Field Theory (Cambridge University Press, Cambridge, 1992), Vol. 2, p. 738.

[32] N. H. Christ, R. Friedberg, and T. D. Lee, Nucl. Phys. B 202, 89 (1982); Nucl. Phys. B 210, 310 (1982); 210, 337 (1982). 
Table I: The values of metric factors $D_{1}, D_{2}^{b}, D_{3}^{b}, D_{2}^{s}$ and $D_{3}^{s}$, for square lattices, random lattices and their duals, with free and periodic boundary conditions in vertical and horizontal directions, respectively.

\begin{tabular}{cccc}
\hline \hline Lattices & Square & Planar random & Dual of planar random \\
\hline$D_{1}$ & 1 & $1.166 \pm 0.020$ & $1.177 \pm 0.016$ \\
$D_{2}^{b}$ & 1 & $1.164 \pm 0.014$ & $1.176 \pm 0.015$ \\
$D_{3}^{b}$ & 1 & $1.512 \pm 0.008$ & $0.778 \pm 0.002$ \\
$D_{2}^{s}$ & 1 & $1.186 \pm 0.012$ & $1.180 \pm 0.014$ \\
$D_{3}^{s}$ & 1 & $1.062 \pm 0.001$ & $1.005 \pm 0.002$ \\
\hline
\end{tabular}




\section{FIGURES}

FIG. 1. Examples of (a) $L_{1} \times L_{2}=8 \times 4$ planar random lattice (solid lines), with its dual (dashed lines) on $L_{1} \times L_{2}=8 \times 4$ rectangular area, with periodic boundary conditions, and (b) a first kind of percolating cluster path, without boundary bonds (bold solid lines) on random lattice.

FIG. 2. $W_{n}\left(L_{1}, L_{2}, p\right)$ on square lattices, planar random lattices and their duals of size $128 \times 32,256 \times 64$, and $512 \times 128$, which are represented by dotted, dashed, and solid lines, respectively.

FIG. 3. (a) $P^{b}$ and $<c^{b}>_{n}$, and (b) $P^{s}$ and $<c^{s}>_{n}$ on square lattice, planar random lattice and its dual of same size $512 \times 128$.

FIG. 4. At $p=p_{c}$, (a) $f\left(c^{b}\right)$ and $f_{n}\left(c^{b}\right)$, and (b) $f\left(c^{s}\right)$ and $f_{n}\left(c^{s}\right)$ on square lattice, planar random lattice and its dual of same size $512 \times 128$.

FIG. 5. The scaled results of $F_{n}(r, y)=W_{n}\left(L_{1}, L_{2}, p\right)$ as a function of $y=\left(p-p_{c}\right) L^{1 / \nu}$ for (a) planar random lattices and (b) their duals of size $128 \times 32,256 \times 64$, and $512 \times 128$. The monotonic decreasing function is for $F_{0}(r, y)$. The $\mathrm{S}$ shaped curve is for $F_{1}(r, y)$. The bell shaped curves from top to bottom are for $F_{n}(r, y)$, with $n$ as 2,3 , and 4 , respectively.

FIG. 6. The scaled results of $U_{n}(x)=W_{n}\left(L_{1}, L_{2}, p\right)$, as a function of $x=D_{1}\left(p-p_{c}\right) L^{1 / \nu}$ for square lattice (solid curves), planar random lattice (dashed curves), and the dual of planar random lattice (dotted curves) of same size $512 \times 128$.

FIG. 7. $U_{n}(r, x)$ for square lattice (solid curves), planar random lattice (dashed curves), and the dual of planar random lattice (dotted curves). (a) $n=1$, the intersection of the curves on $x=0$ axis, from up to down are for $r=L_{1} / L_{2}=1,2,3, \ldots, 6$. (b) The width of curves, from small to large are for $r=L_{1} / L_{2}=1,2,3, \ldots, 6$.

FIG. 8. (a) $U_{n}(r, 0)$ as a function of $r=L_{1} / L_{2}$, for a number of percolating clusters (npc) run from 0 to 4 , and (b) $C(r, 0)$ as a function of $r=L_{1} / L_{2}$, with slope of the fitting line 0.43. Square lattice $(\square)$, planar random lattice $(\triangle)$ and the dual of planar random lattice 
$(\times)$, all have horizontal periodic boundary conditions.

FIG. 9. The scaled results of $P(p, L) / L^{-\beta / \nu}$, in terms of bonds $\left(P=P^{b}\right)$ and sites $\left(P=P^{s}\right)$, for different types of lattice size $256 \times 64$ and $512 \times 128$, as a function of $y=\left(p-p_{c}\right) L^{1 / \nu}$.

FIG. 10. The scaled results of $G_{n}(z, L)=D_{3}<c(p, L)>_{n} / L^{-\beta / \nu}$, as a function of $z=$ $D_{2}\left(p-p_{c}\right) L^{1 / \nu}$, for square lattices, planar random lattices and their duals of same size $256 \times 64$, and $512 \times 128$. (a) $c=c^{b}, D_{2}=D_{2}^{b}, D_{3}=D_{3}^{b}$, and (b) $c=c^{s}, D_{2}=D_{2}^{s}, D_{3}=D_{3}^{s}$.

FIG. 11. The scaled results of $H_{n}\left(z^{\prime}, L\right)=D_{3}^{-1} f_{n}(c) / L^{\beta / \nu}$, as a function of $z^{\prime}=D_{3}(p-$ $\left.p_{c}\right) L^{\beta / \nu}$ for square lattices, planar random lattices, their duals of same size $256 \times 64$, and $512 \times 128$, with fitting results (solid curves); (a) $c=c^{b}, D_{3}=D_{3}^{b}$, and (b) $c=c^{s}, D_{3}=D_{3}^{s}$. 
(a)

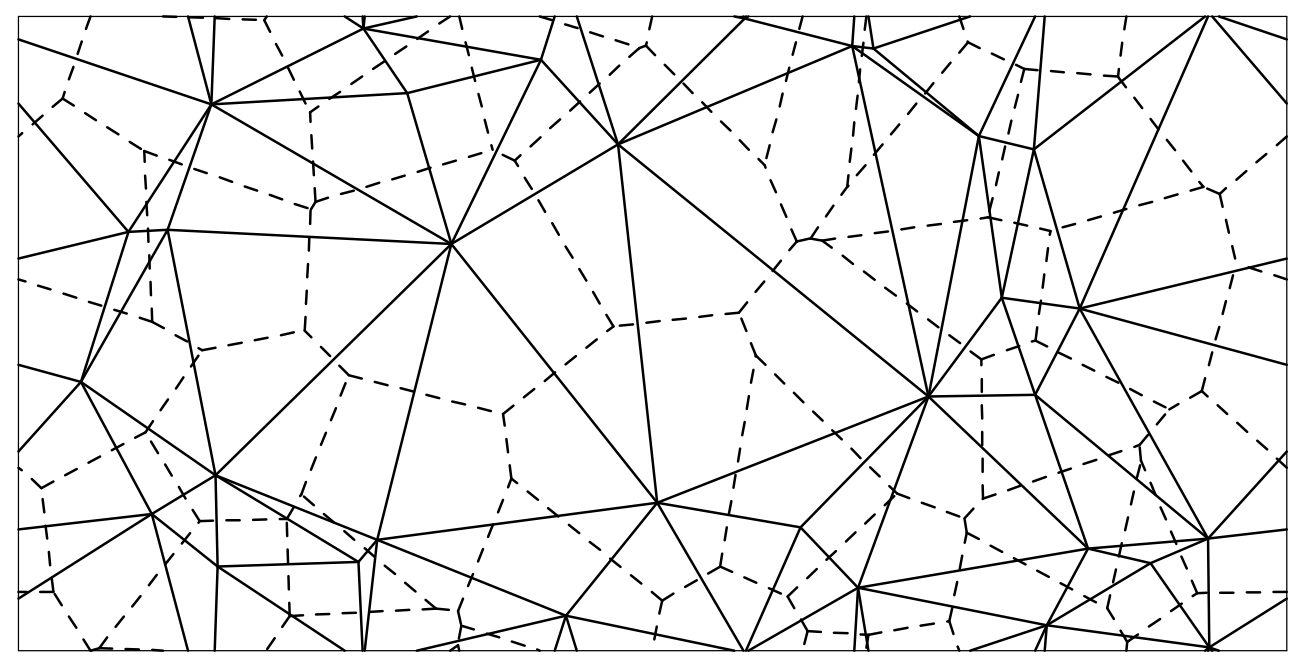

Fig. 1 (a) Hsu, Lin and $\mathrm{Hu}$ 
(b)

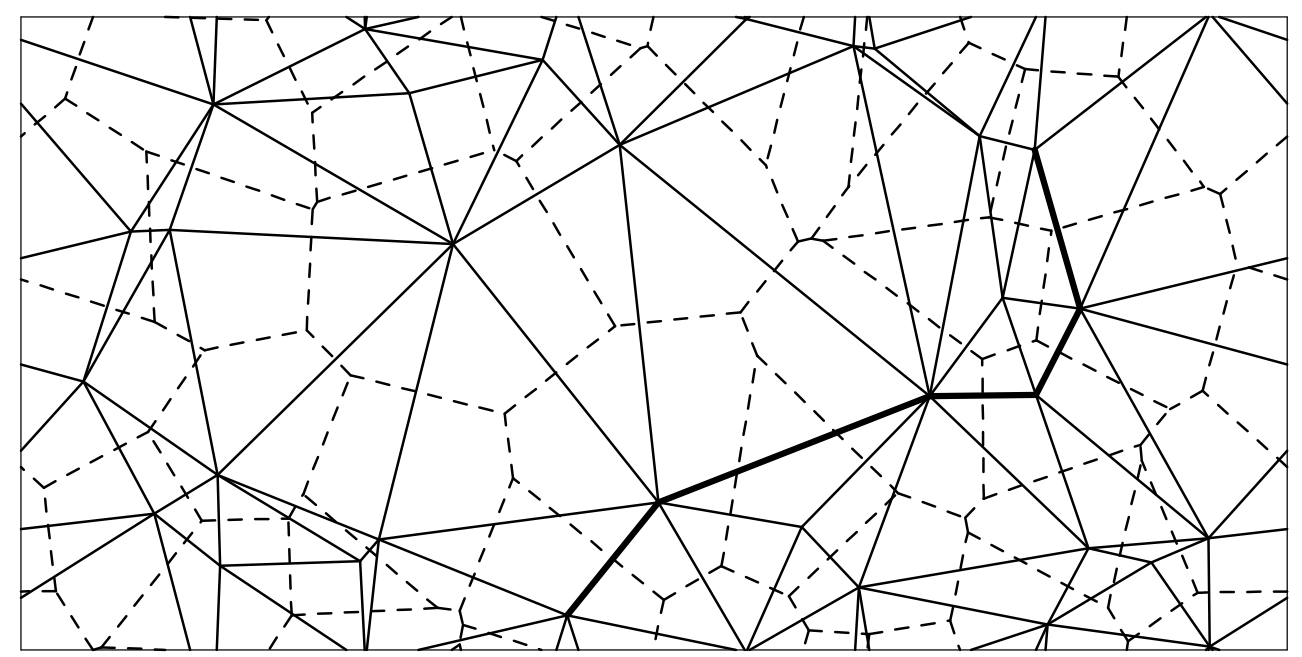

Fig. 1 (b) Hsu, Lin and $\mathrm{Hu}$ 


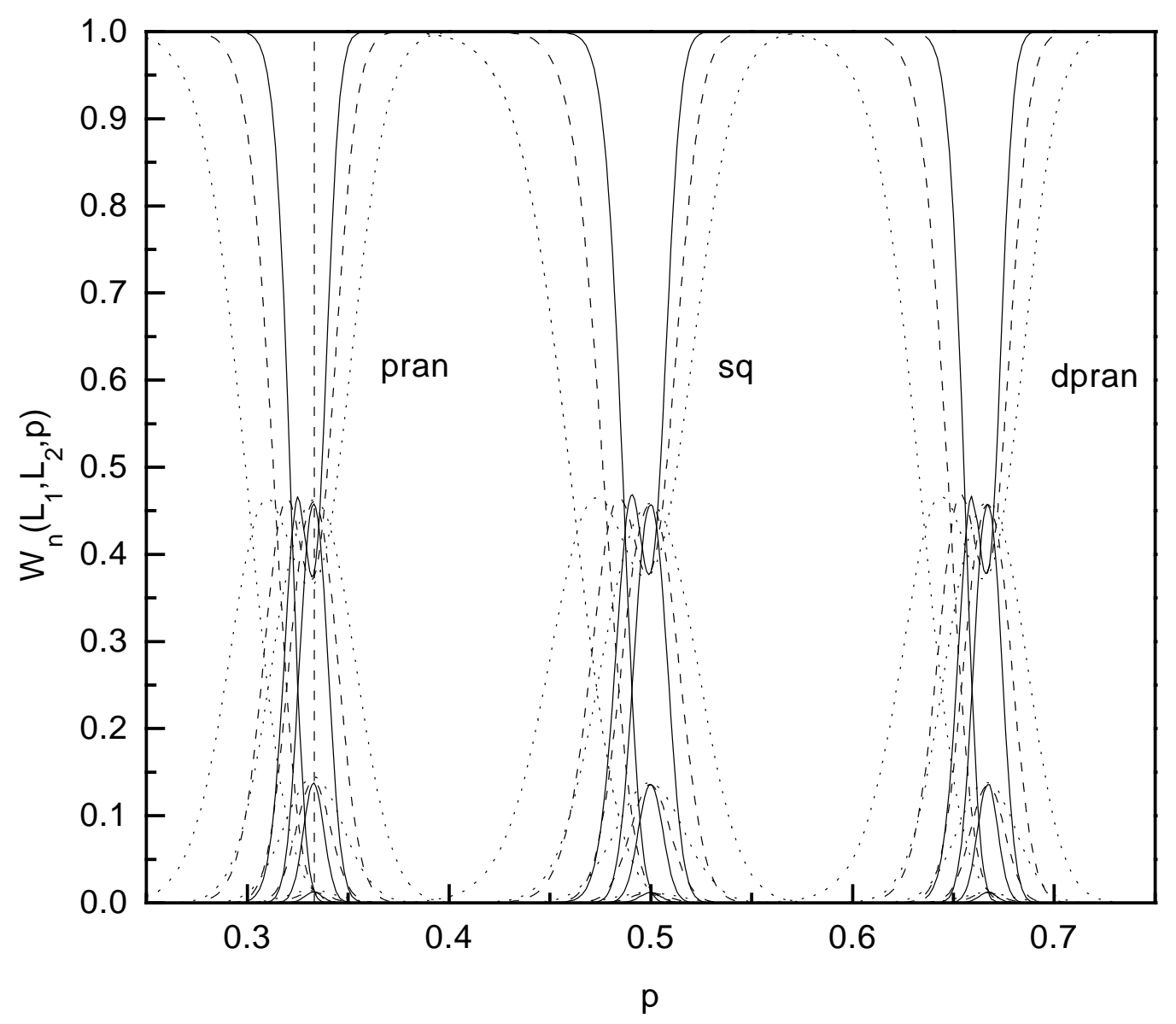

Fig. $2 \mathrm{Hsu}$, Lin and $\mathrm{Hu}$ 


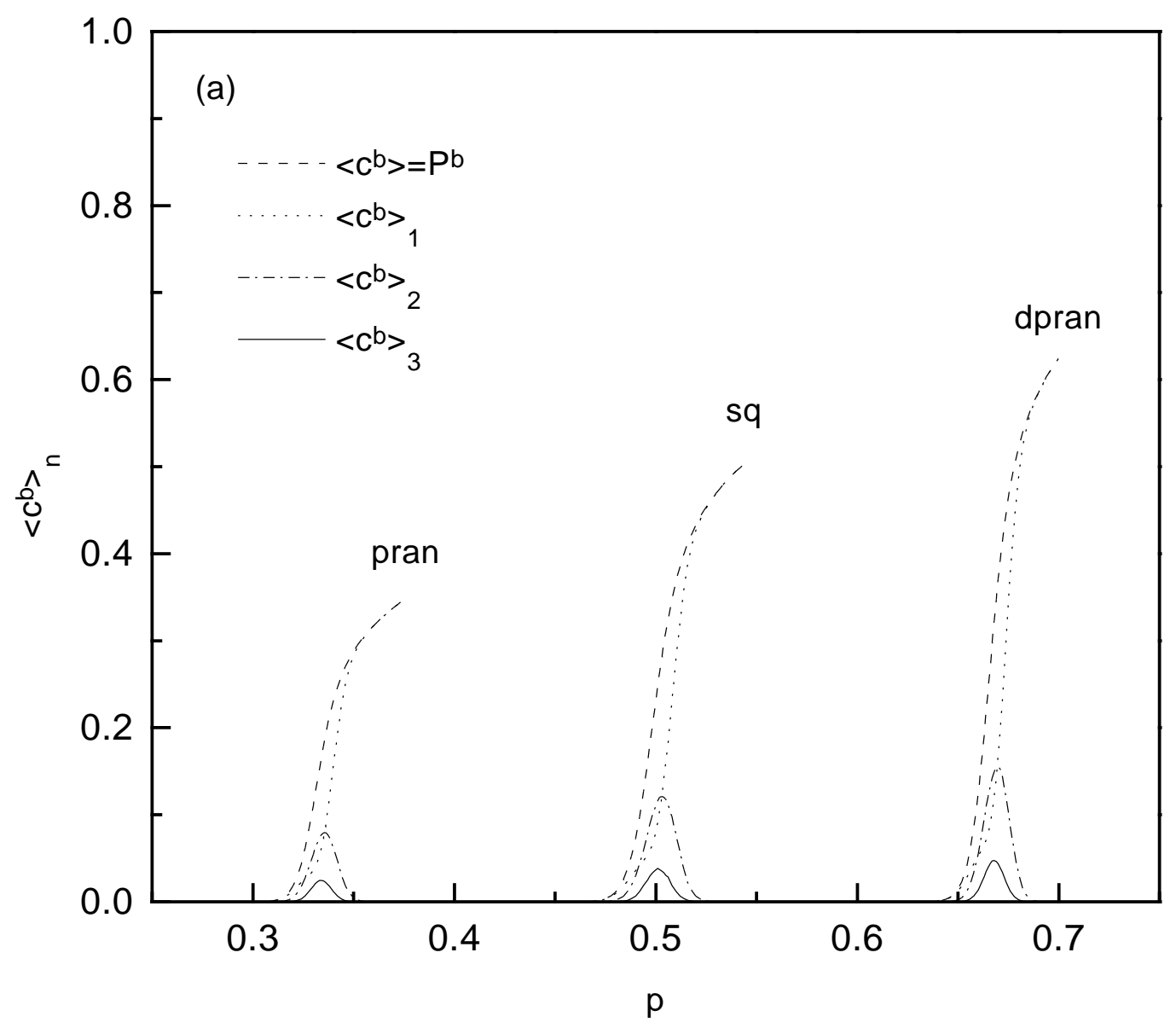

Fig. 3(a) Hsu, Lin and $\mathrm{Hu}$ 


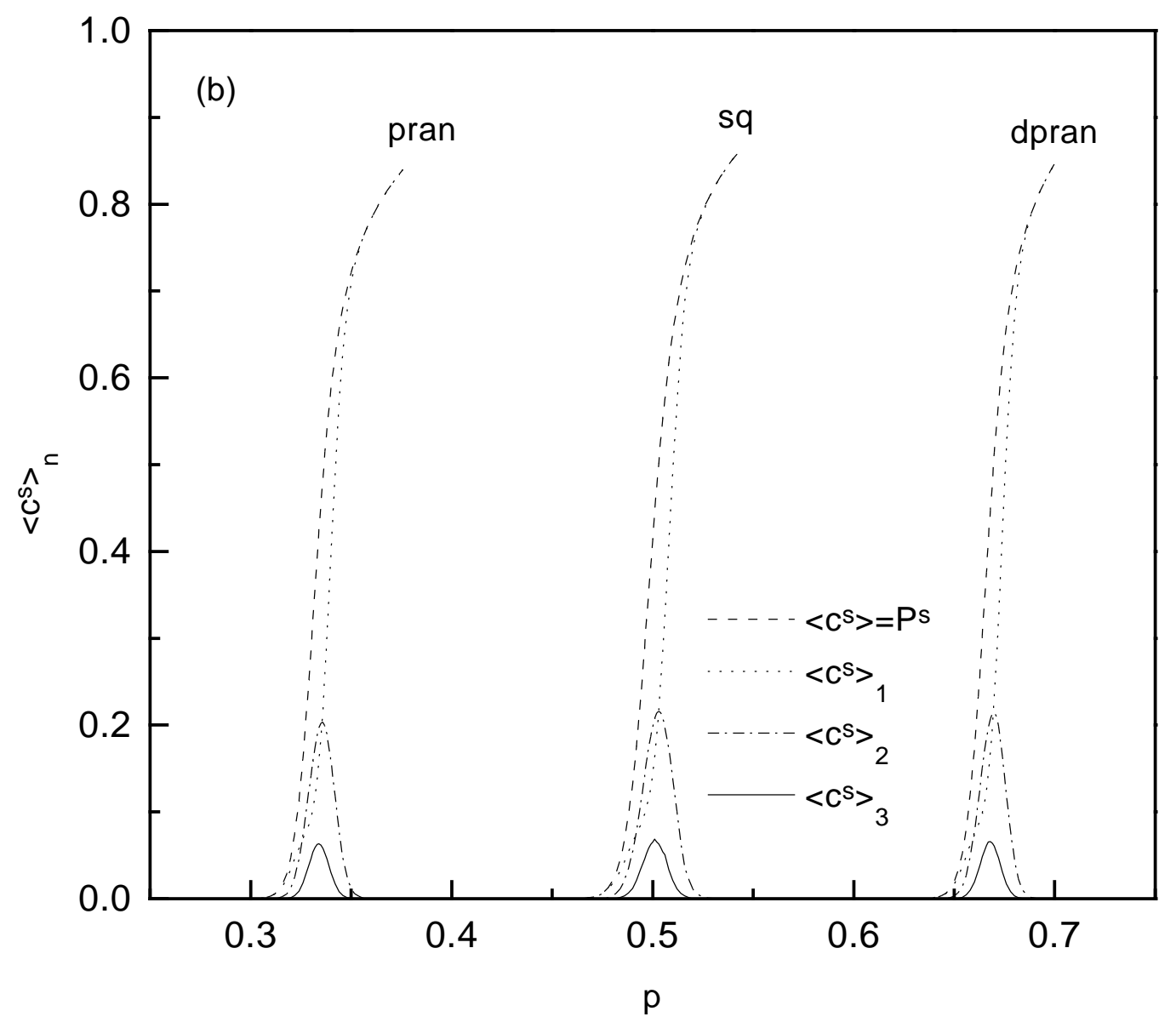

Fig. 3(b) Hsu, Lin and $\mathrm{Hu}$ 


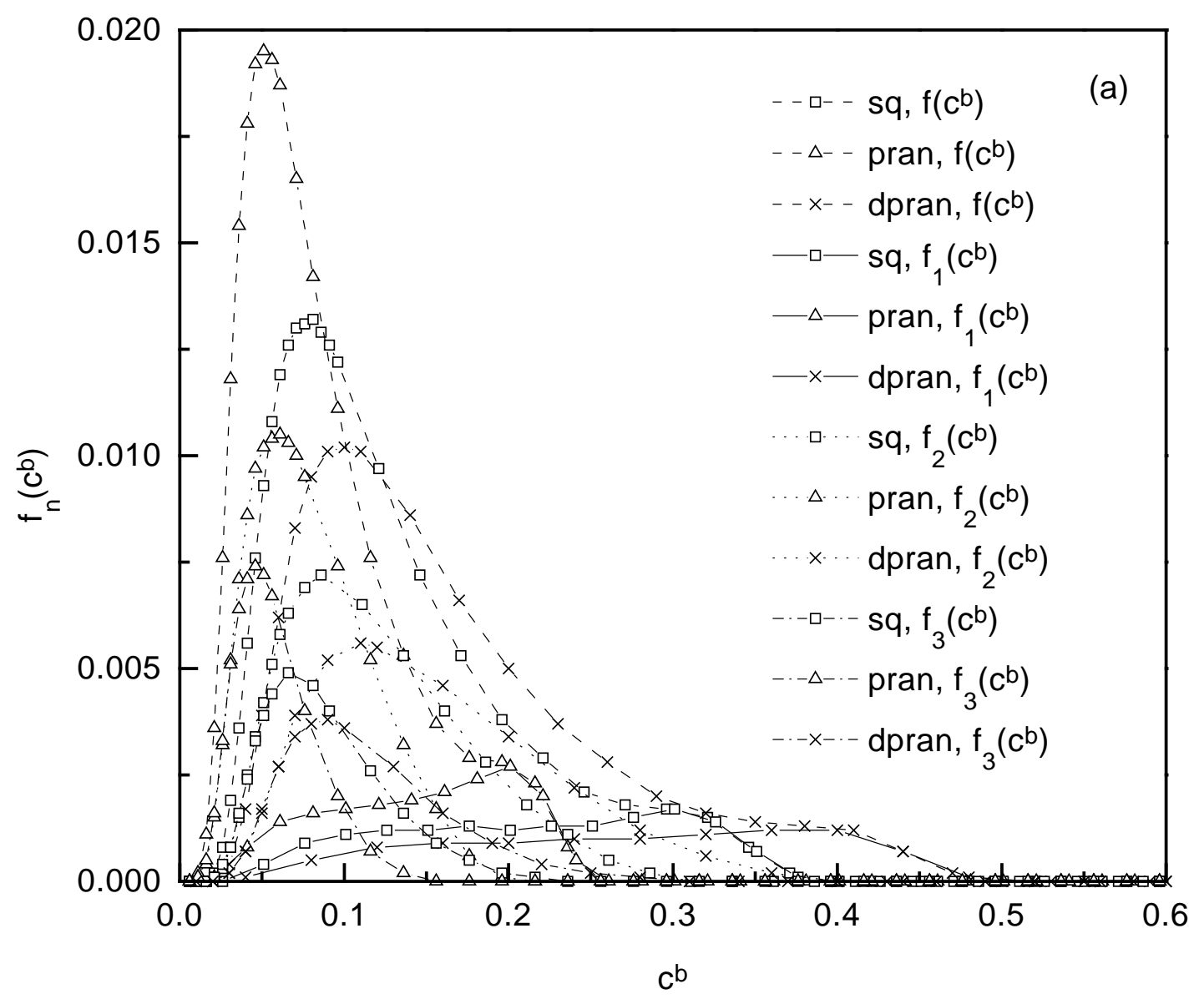

Fig. 4(a) Hsu, Lin and $\mathrm{Hu}$ 


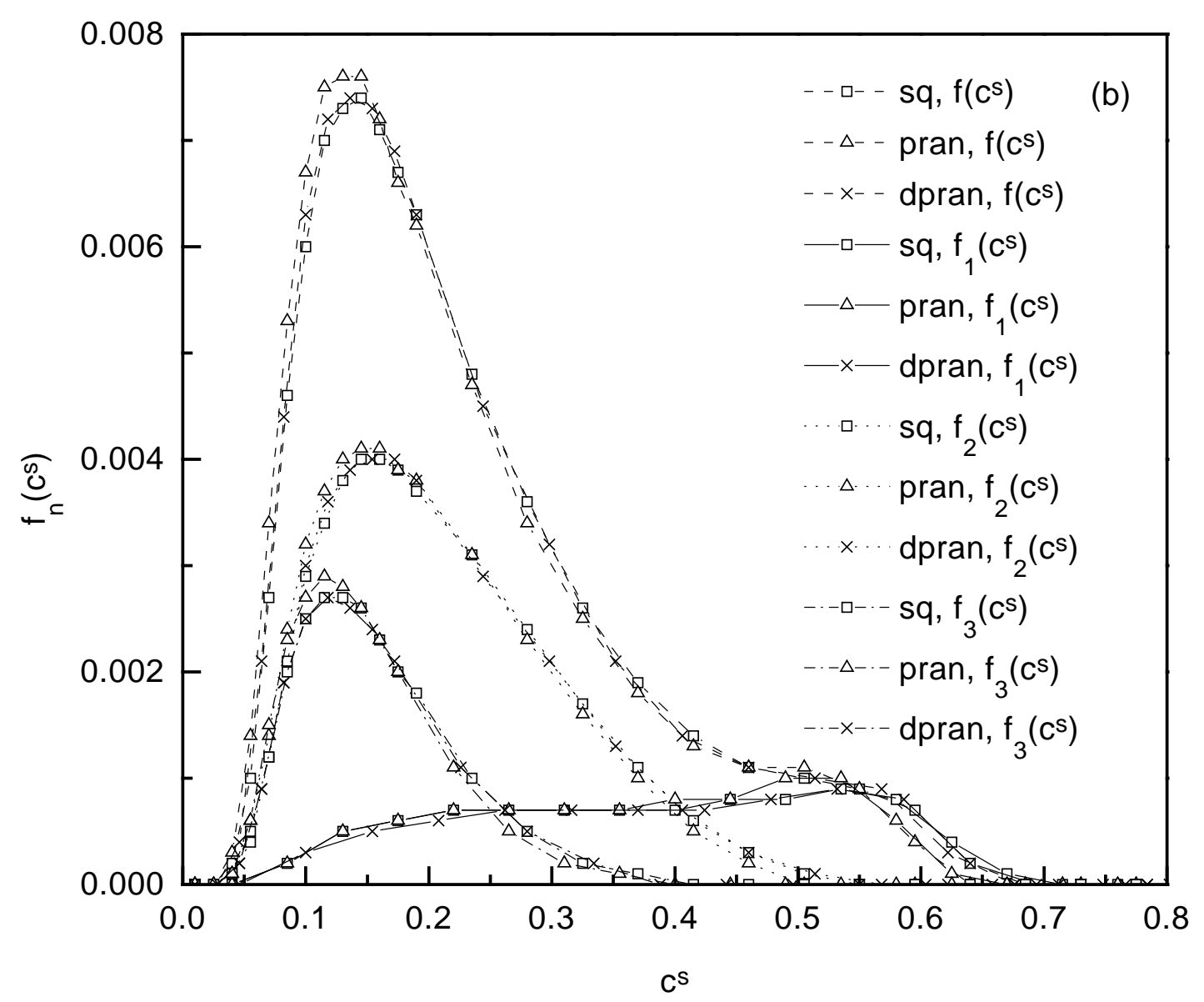

Fig. 4(b) Hsu, Lin and Hu 


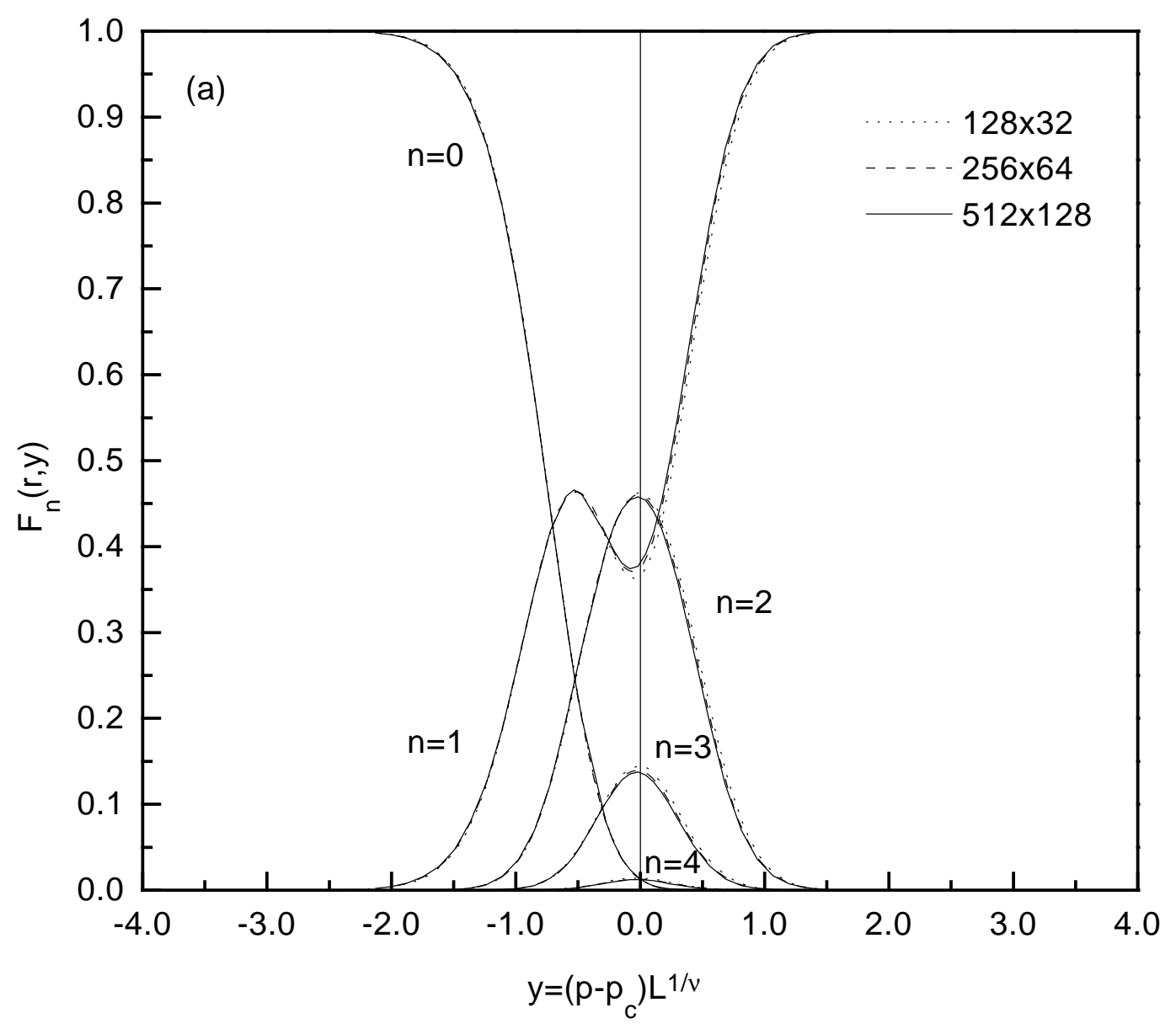

Fig. 5(a) Hsu, Lin and $\mathrm{Hu}$ 


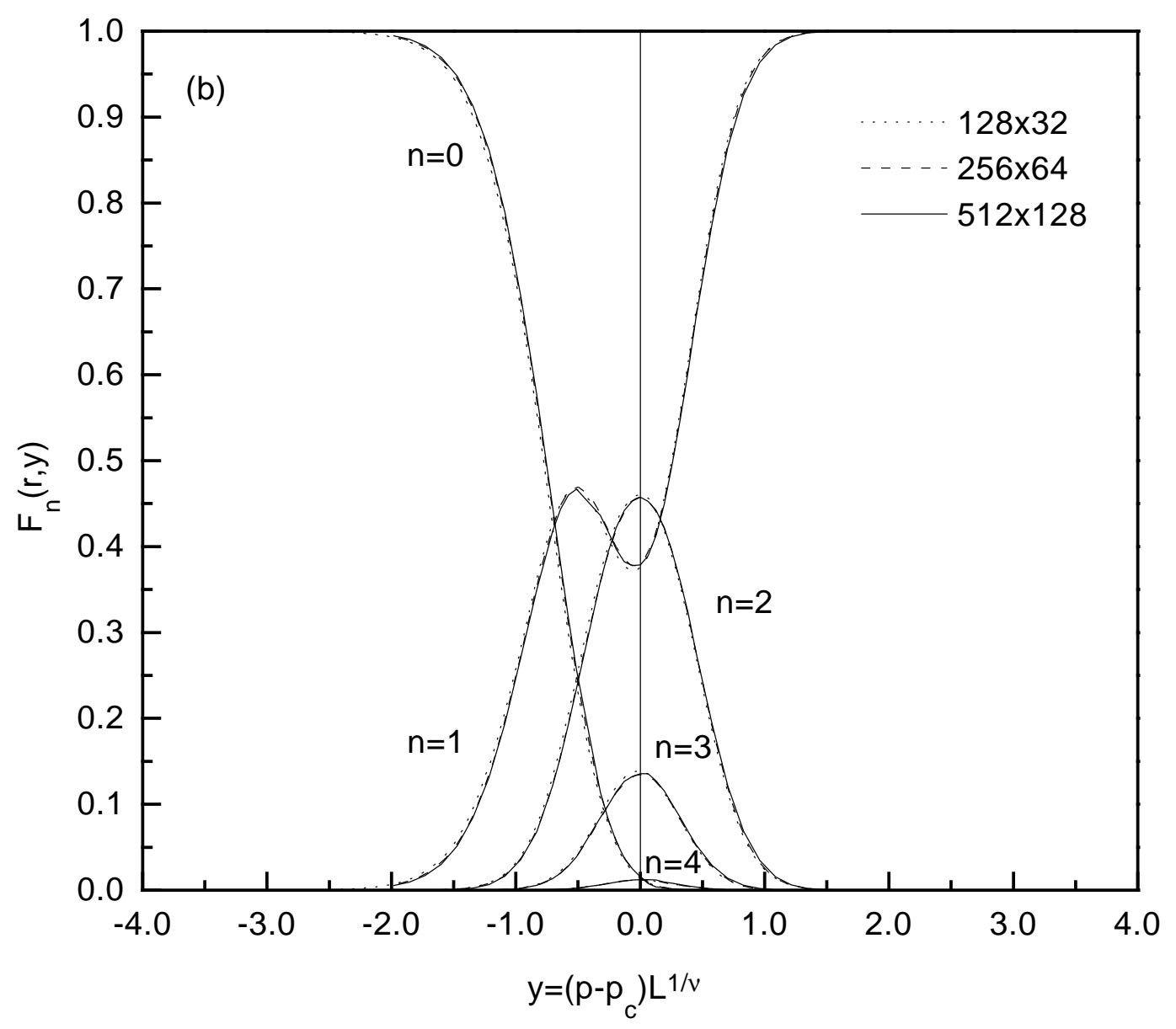

Fig. 5(b) Hsu, Lin and Hu 


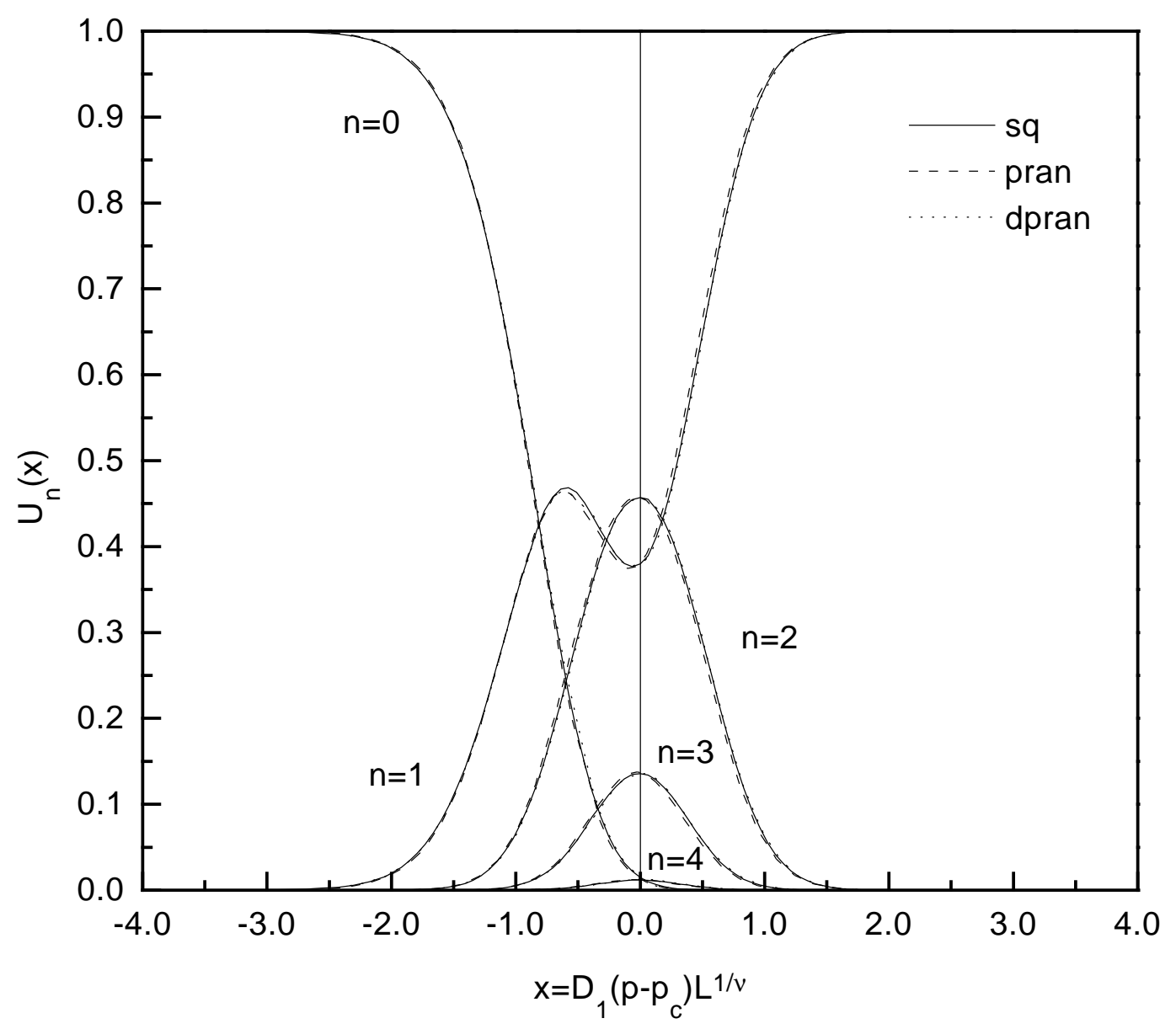

Fig. $6 \mathrm{Hsu}$, Lin and $\mathrm{Hu}$ 


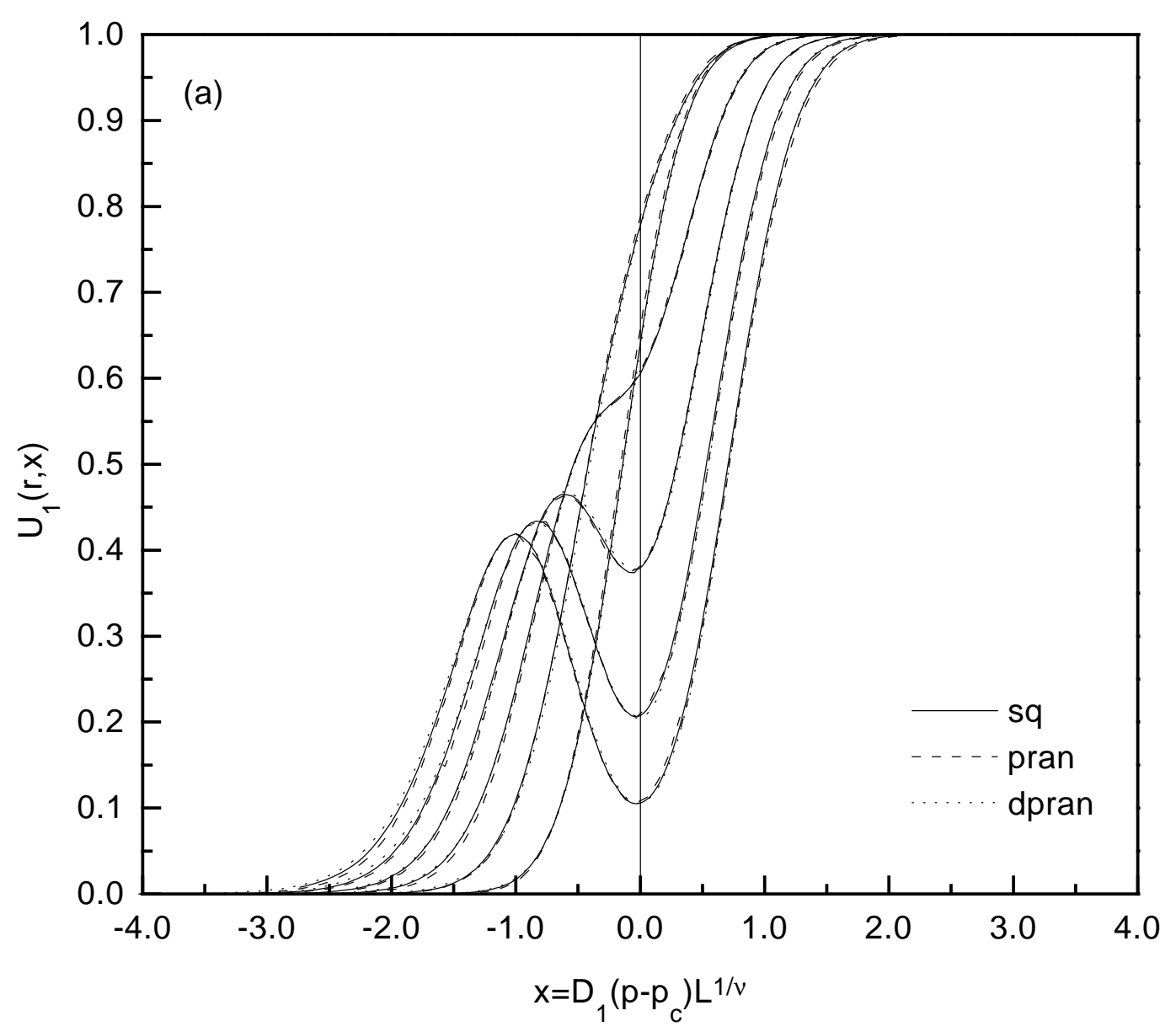

Fig. 7(a) Hsu, Lin and $\mathrm{Hu}$ 


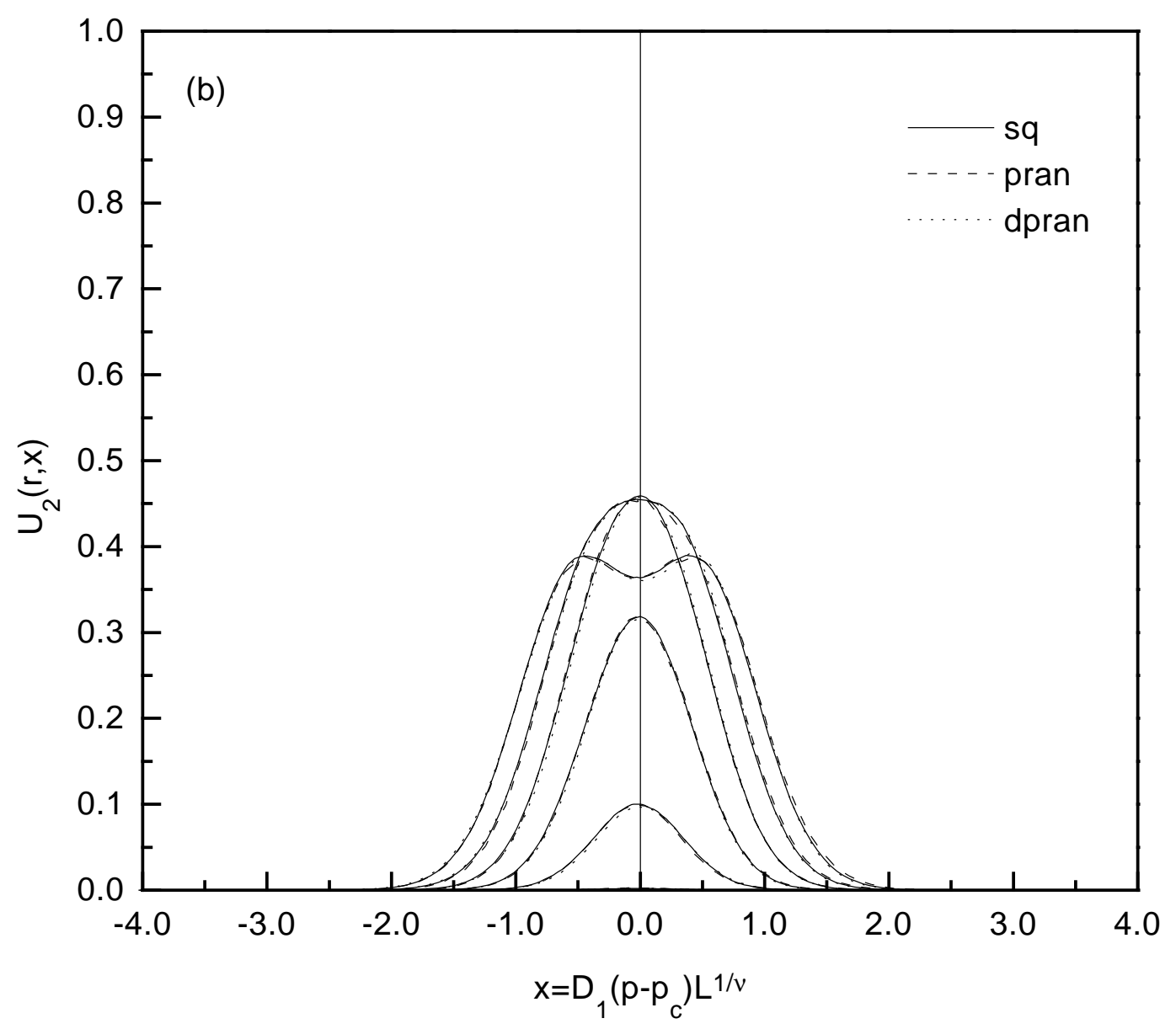

Fig. 7(b) Hsu, Lin and $\mathrm{Hu}$ 


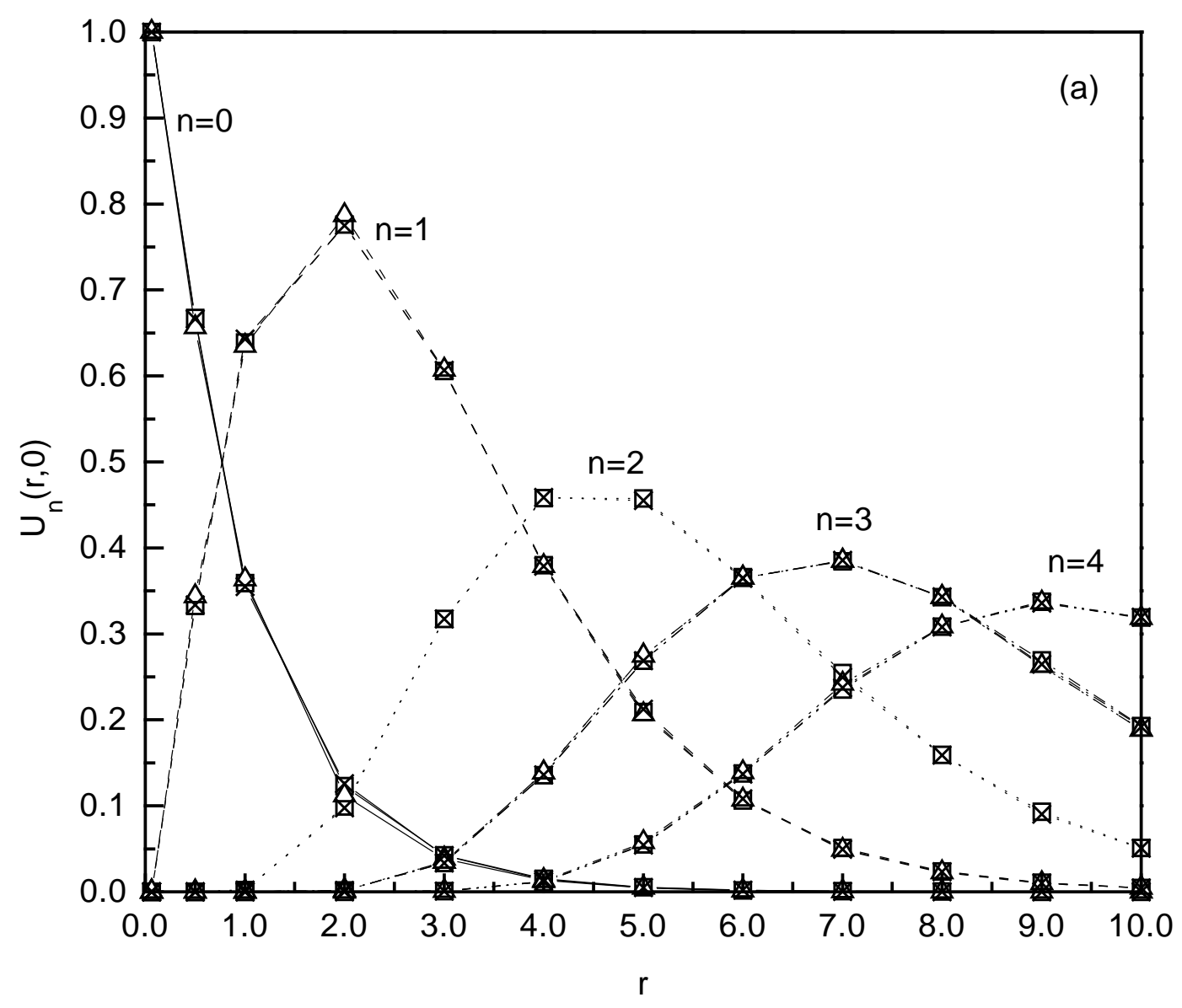

Fig. 8(a) Hsu, Lin and $\mathrm{Hu}$ 


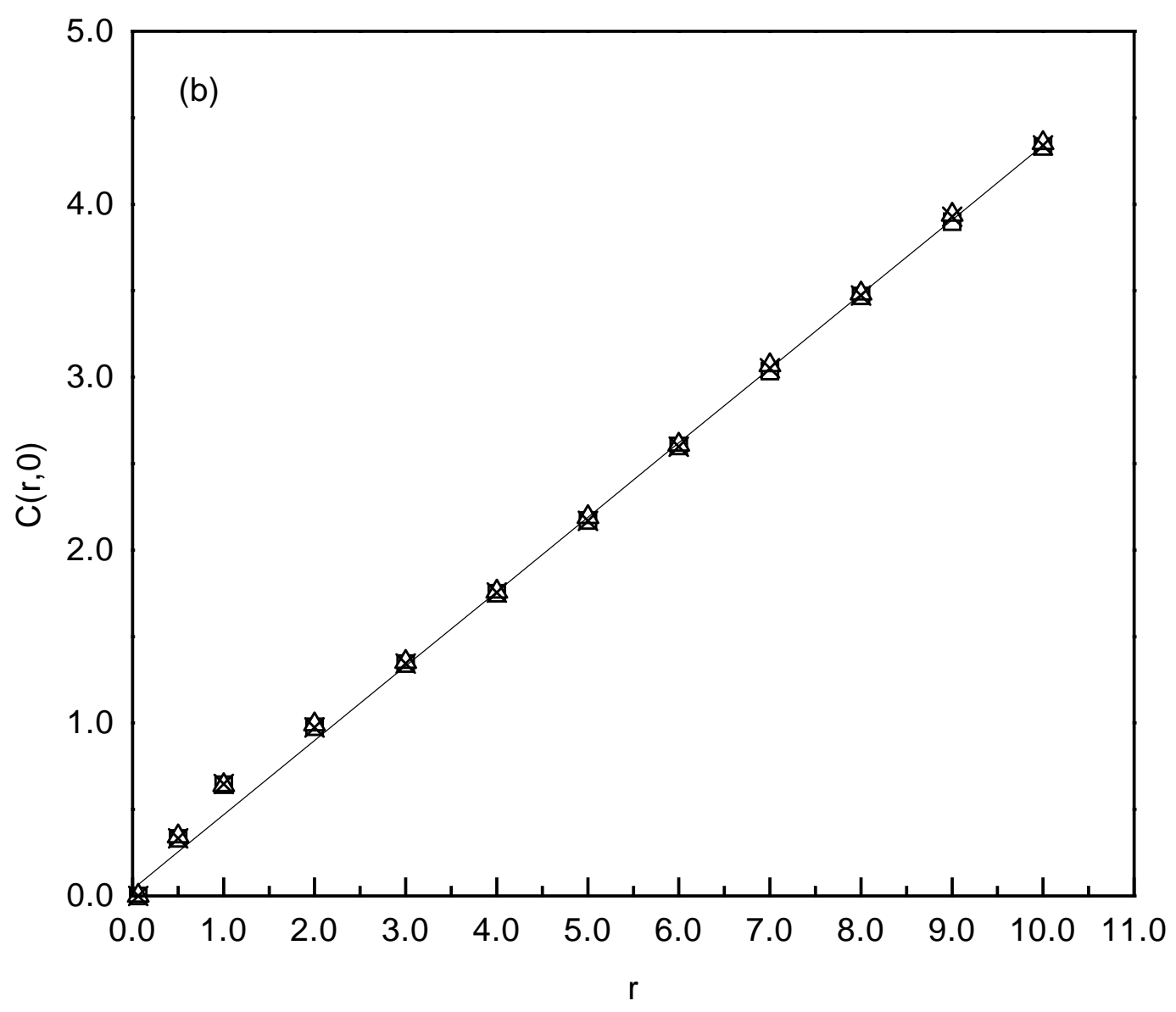

Fig. 8(b) Hsu, Lin and $\mathrm{Hu}$ 


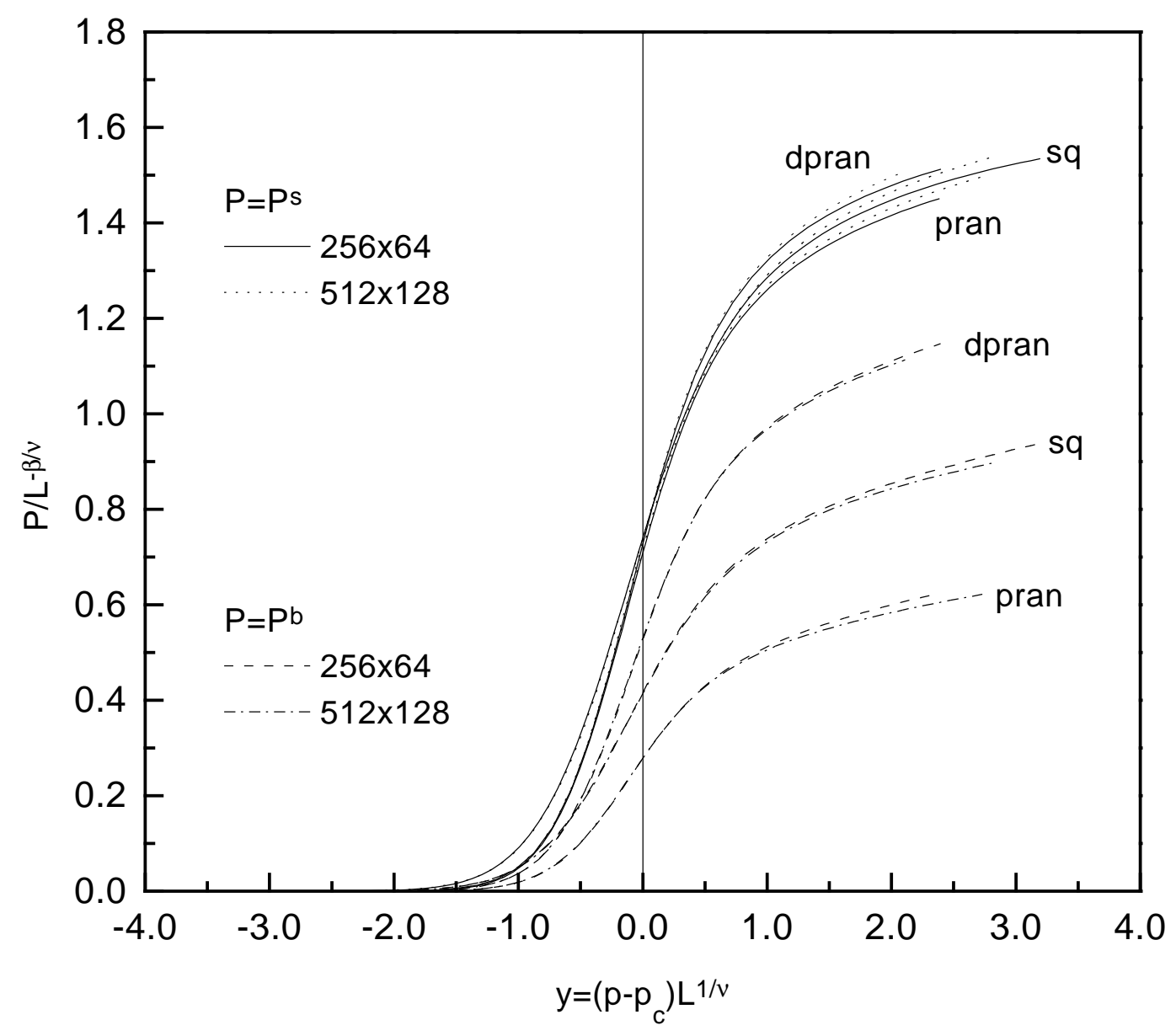

Fig. $9 \mathrm{Hsu}$, Lin and $\mathrm{Hu}$ 


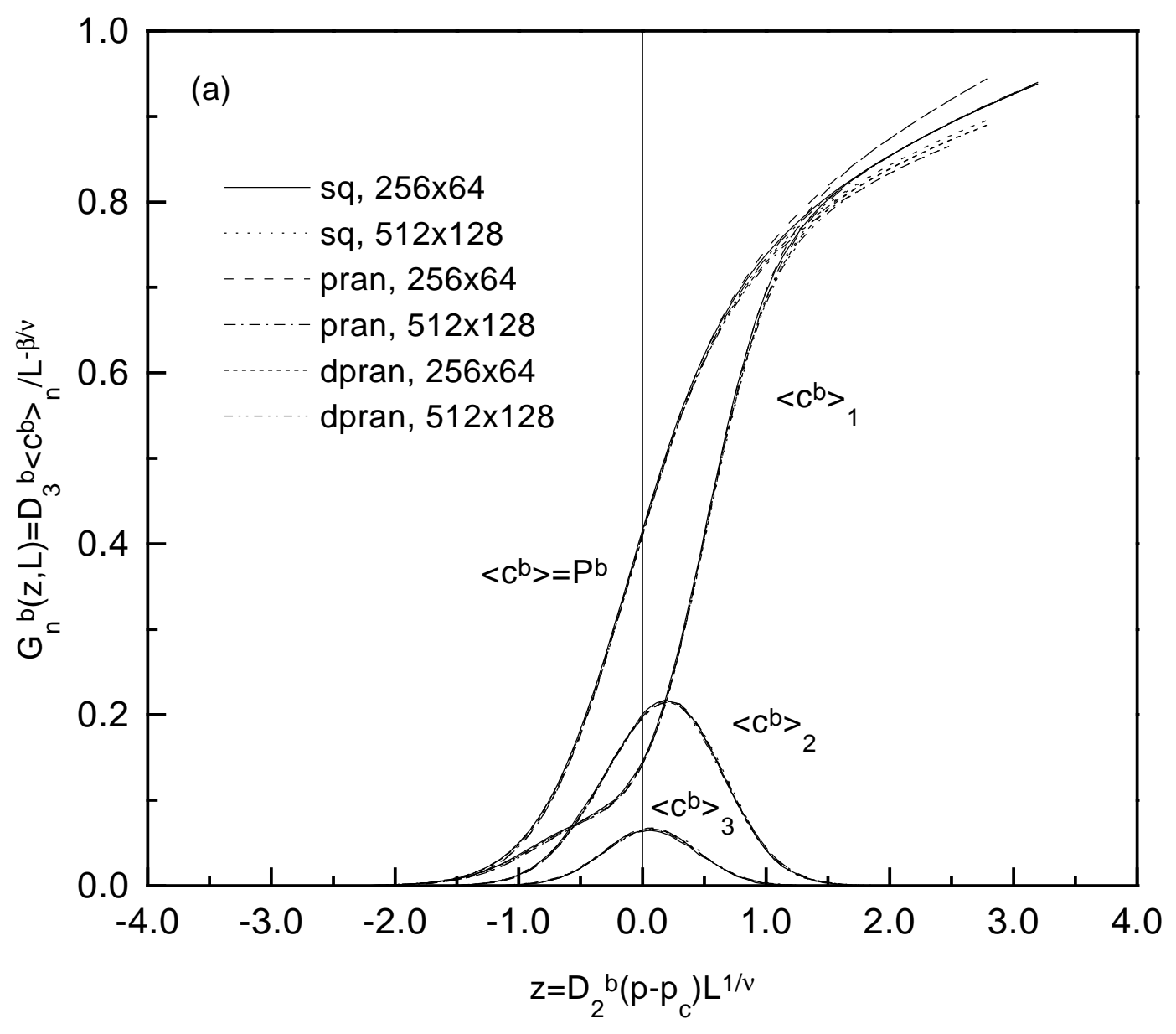

Fig. 10(a) Hsu, Lin and $\mathrm{Hu}$ 


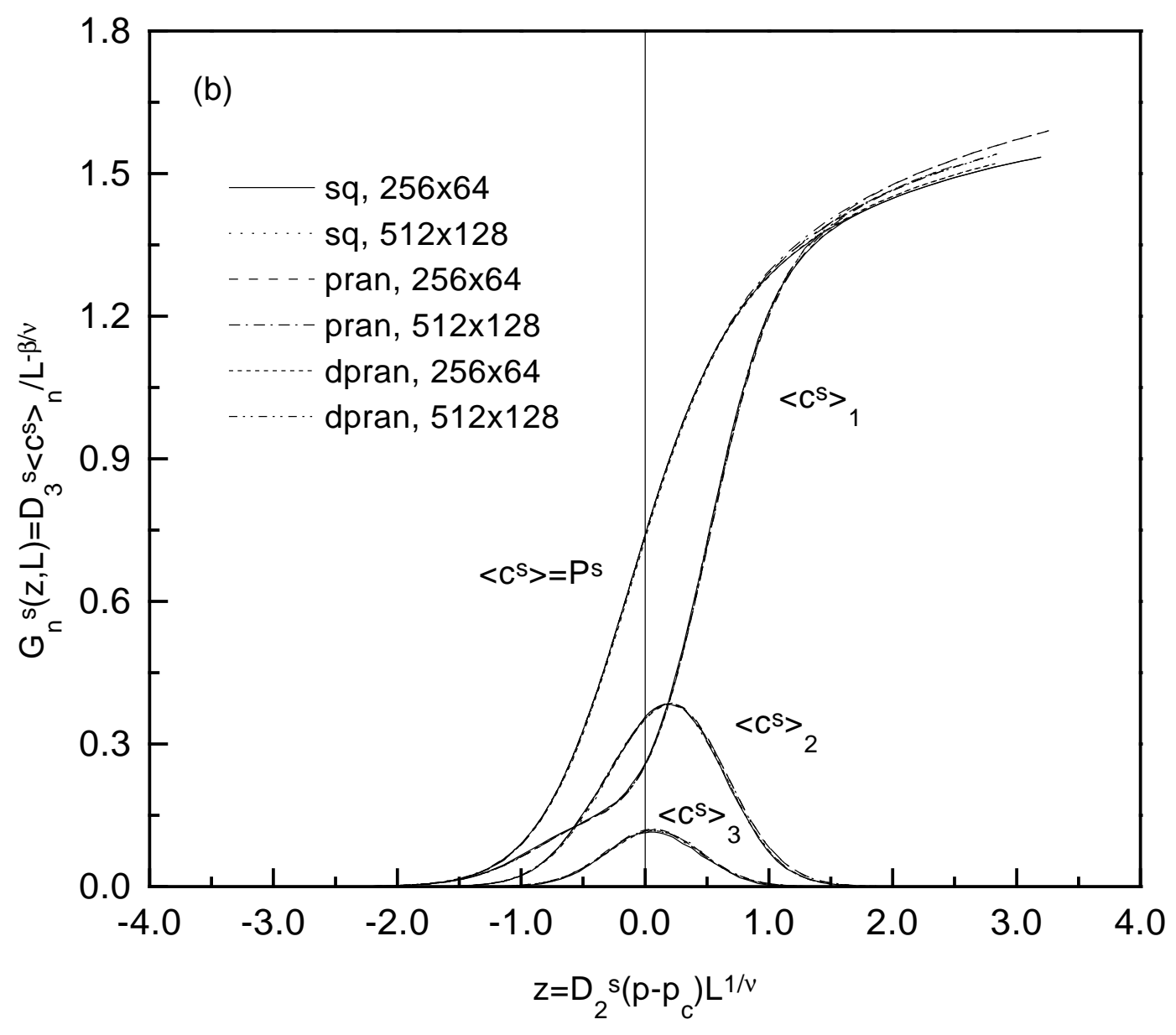

Fig. 10(b) Hsu, Lin and Hu 


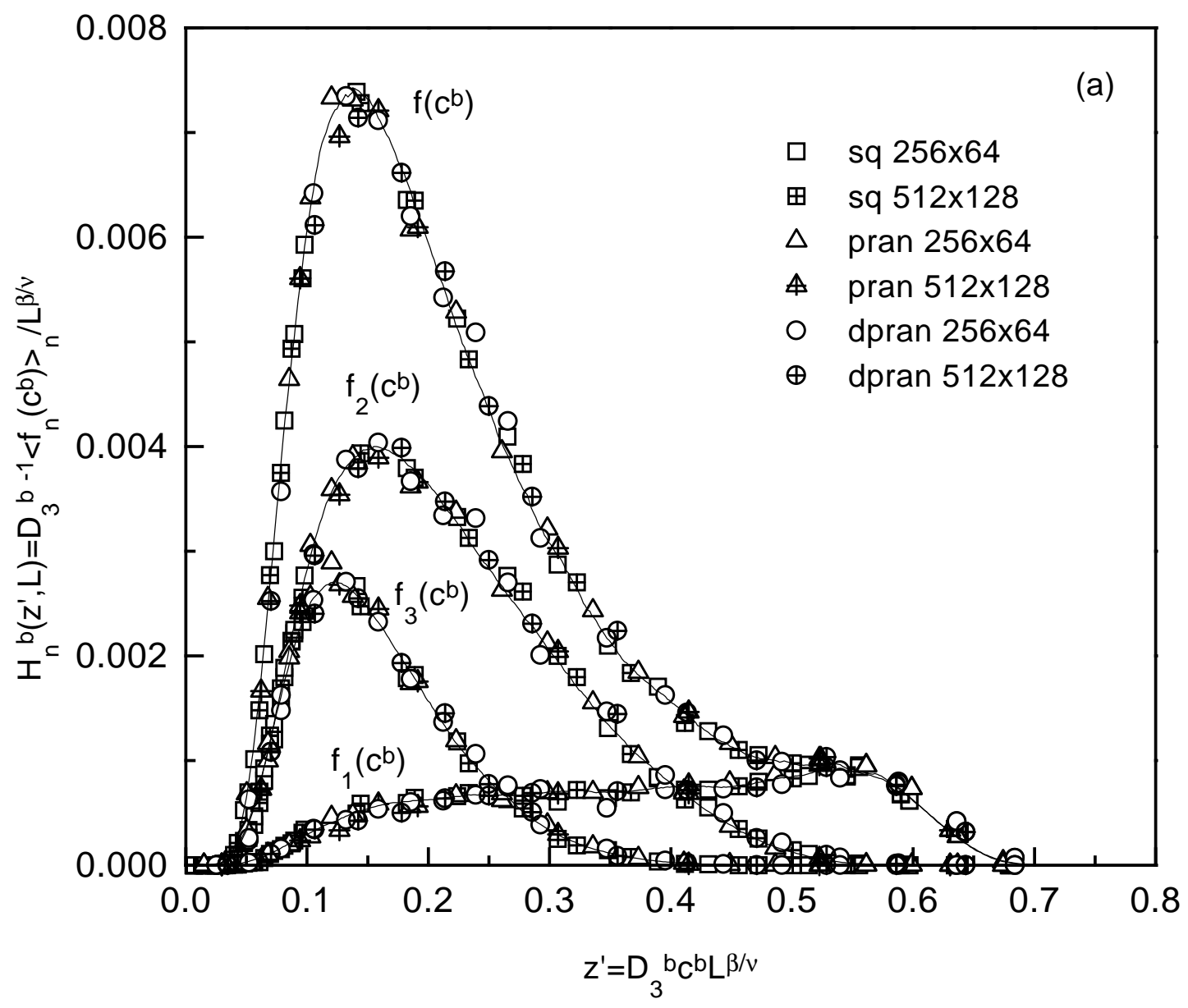

Fig. 11(a) Hsu, Lin and $\mathrm{Hu}$ 


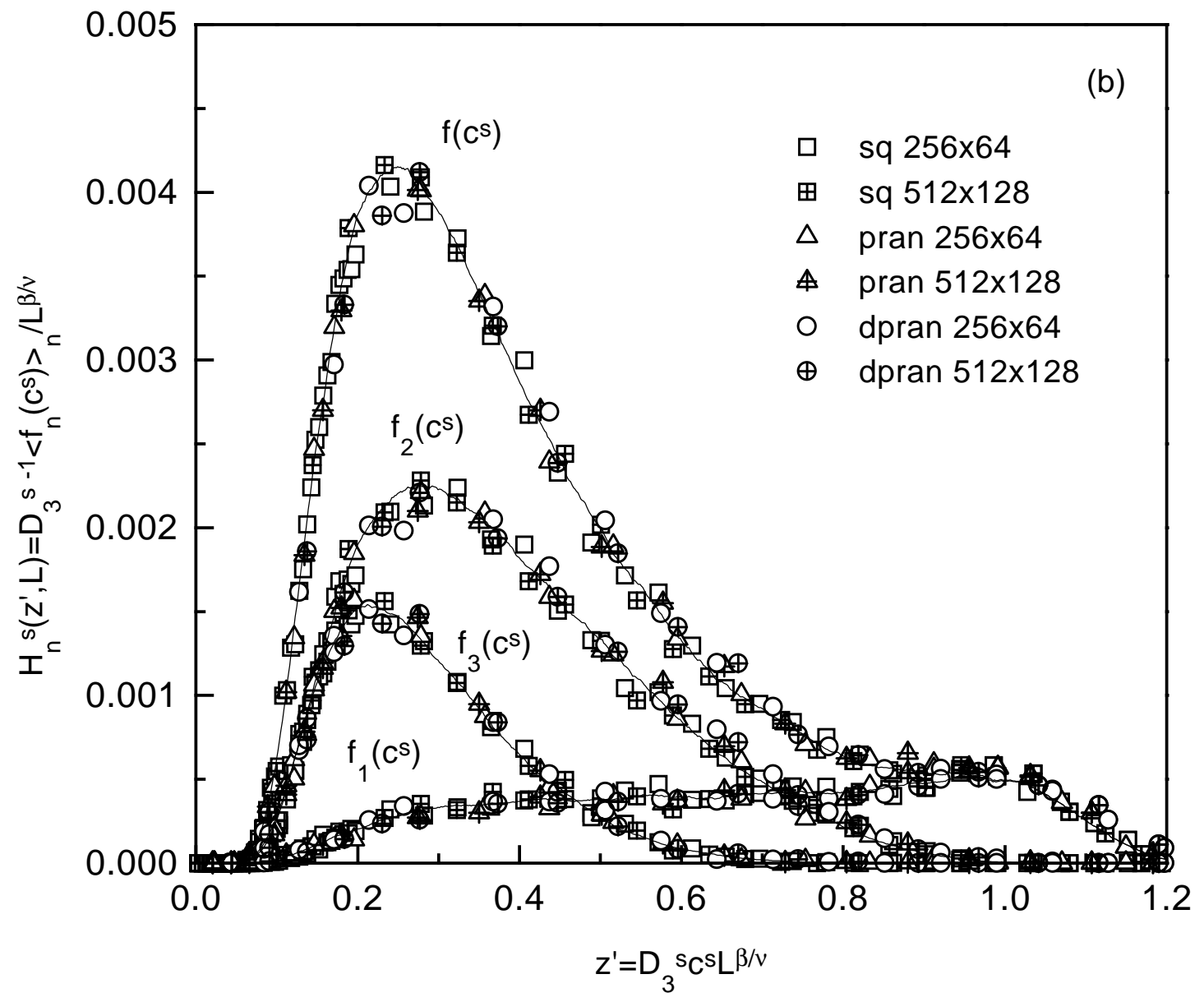

Fig. 11(b) Hsu, Lin and $\mathrm{Hu}$ 